\title{
Fuzzy Urban Sets \\ Theory and Application to \\ Desakota Regions in China
}

July 2002

by

Eric J. Heikkila, Associate Professor*

School of Policy, Planning, and Development

University of Southern California

Los Angeles, CA 90089-0626

Ti-yan Shen, Lecturer

School of Government

Peking University

Beijing, China

\author{
Kai-zhong Yang, Professor and Vice-Dean \\ School of Government \\ Peking University \\ Beijing, China
}

\begin{abstract}
:
This paper outlines a method for using the mathematics of fuzzy sets that is well suited to measure and characterize peri-urbanizing ("desakota") systems typical of China, Southeast Asia, and other areas experiencing rapid urbanization. Drawing on Kosko's "fuzzy hypercube", three distinct but interdependent measures are derived: (i) extent of urbanization, (ii) level of fuzziness, and (iii) degree of entropy. The feasibility of the proposed method is demonstrated using remote sensing data for Ningbo, China.

* The research presented here was undertaken in part while Eric Heikkila was a Visiting Fullbright Scholar at Peking University, and support from the Council for International Exchange of Scholars is gratefully acknowledged. We are also grateful for supplementary funding from USC's Center for International Studies and the USC Lusk Center for Real Estate to support additional collaboration on this research vital to finalizing this draft. Bart Kosko of USC, Terry McGee of University of the British Columbia, and Anthony Yeh of the University of Hong Kong provided valuable feedback on an earlier draft of this paper, as did participants at seminars organized respectively by the Department of Geography at Hong Kong Baptist University, the Department of Geography at Nanjing University, and the Tokyo Development Forum. Wu Bo provided insightful computer programming assistance. Finally, we are grateful to Michael Batty and three anonymous referees for helpful suggestions. We fully retain the usual responsibilities of authorship. All correspondence should be addressed to first author.
\end{abstract}

forthcoming 2002, Environment \& Planning B: Planning and Design, vol. 29 


\section{Introduction}

The desakota land use pattern that is characteristic of Southeast Asia, China, and other rapidly urbanizing regions is notable for its urban-rural ambiguity. Figure 1 re-presents the classic depiction of this peri-urbanization pattern as introduced by Terry McGee (1991). While much work has been done by urban geographers to understand and to document this phenomenon, less has been accomplished by way of systematic measurement of peri-urbanization. Indeed, the very nature of the phenomenon defies ready categorization and measurement and renders conventional measures obsolete.

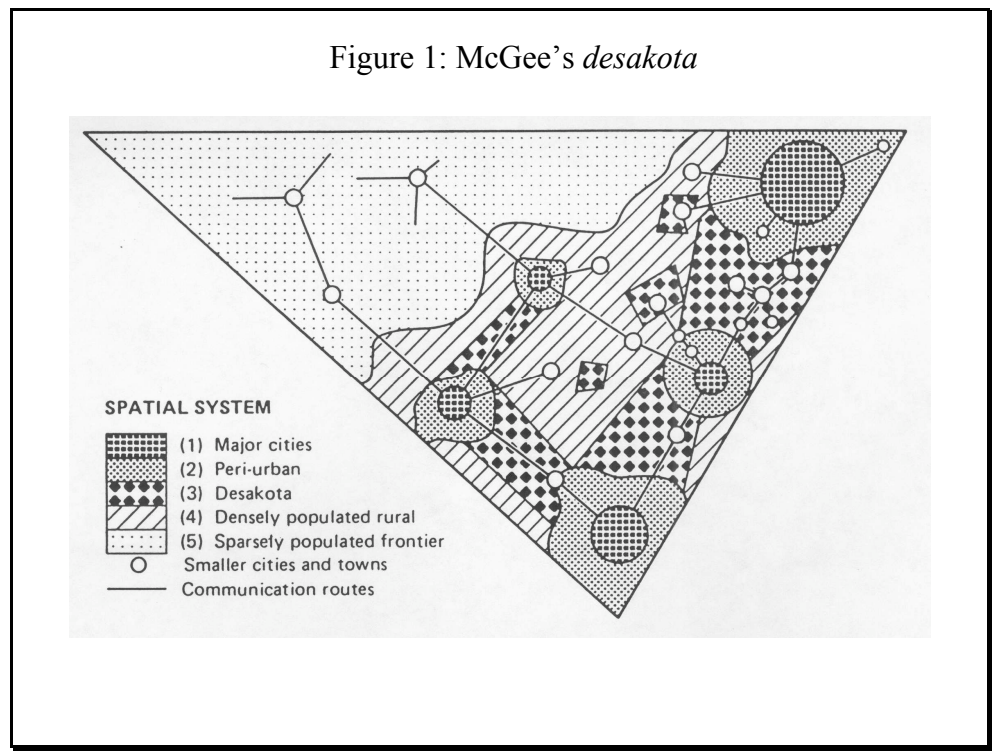

This paper addresses the measurement issue by drawing on the mathematical formulation of fuzzy sets. A fuzzy set is one for which the degree of membership for any element of the set may range from zero to one, and so is well suited to ambiguous or partial membership. In our context we are interested in fuzzy urban sets, the constituent parcels (or pixels) of which may exhibit varying degrees of inclusion. The fuzzy set formulation is a very natural one for desakota settings, and it is easy to envision, for example, how the degree of membership in the fuzzy urban set $U$ may vary from one location to the next in figure 1 . In contrast, conventional (or "crisp") sets, for which all elements are constrained to have full membership, seem ill-suited to desakota settings, as would be any crisp rendering of a peri-urbanizing territory into two discrete mutually exclusive and non-overlapping subsets, urban and rural. 
The use of fuzzy sets here is consistent with that of fuzzy classification methods that are increasingly used in remote sensing to allow pixels to retain some degree of membership in one or more land use classes (see, for example, Campbell, 1996, Chapter 11). We go further by focusing on the nature of the implied fuzzy urban set itself. Stated another way, fuzzy classification methods focus on the remote sensing technique used to ascertain degrees of membership for each pixel, whereas our focus is on the representation and description of desakota regions as fuzzy sets.

Our approach leads to three distinct yet integrated measures of urbanization for any given study area:

i. extent of urbanization -- the aggregate level of membership in the fuzzy set $U$;

ii. level of fuzziness -- the overall degree of ambiguity regarding membership in $U$;

iii. degree of entropy - uniformity of membership in $U$.

Characterizing (remote sensing images of) urbanizing regions as fuzzy urban sets provides a single integrated perspective from which these three distinct measures are simultaneously defined. As we shall see, previous work by Bart Kosko $(1997,1992)$ provides a convenient and conceptually imaginative framework for this approach.

The next section of this paper indicates the focus of this work in the broader context of research on urbanization in China and elsewhere. Although the proposed method has relevance to other regions, China provides both the motivation for this work and the setting for this particular application. Section three explains the relevance of fuzzy set formulations for characterizing peri-urban systems, and goes on to show how Kosko's $(1997,1992)$ depiction of a fuzzy hypercube lends itself very naturally to the derivation of three relevant measures, all derived from a single conceptual framework. Section four demonstrates the feasibility of the proposed approach using 1987, 1990 and 1996 remote sensing images of Ningbo City, in China's Zhejiang Province. The concluding section discusses theoretical and practical issues that will need to be addressed if the proposed method is to be adapted to regular use.

\section{Broader context of urbanization in China}

Figure 2 depicts the contribution of this paper in the broader context of research on urbanization in China. It is helpful in understanding both what the contribution is and what it is not. There are 
four elements identified in figure 2; each corresponding to a particular facet of urbanization as a field:

[1] China urbanization as a desakota geographic phenomenon

[2] remote sensing images of this phenomenon

[3] summary statistics about urbanization

[4] dynamic models of urbanization

Each of these is an area of study unto itself. Regarding the study of desakota urbanization as a geographic phenomenon [1], McGee's desakota descriptive model has been extended to the Chinese context by Zhou Yixing (1991), Yok-Shiu Lee (1991) and Guldin (1997). Similarly, Zhou Daming (1997) attempts to resolve the urban-rural dichotomy issue by outlining a descriptive model of "rural urbanization in China". The references above focus specifically on documenting and describing the desakota phenomenon in the Chinese context. There is also a voluminous literature addressing other aspects of urbanization in China but that is beyond the scope of our immediate focus.

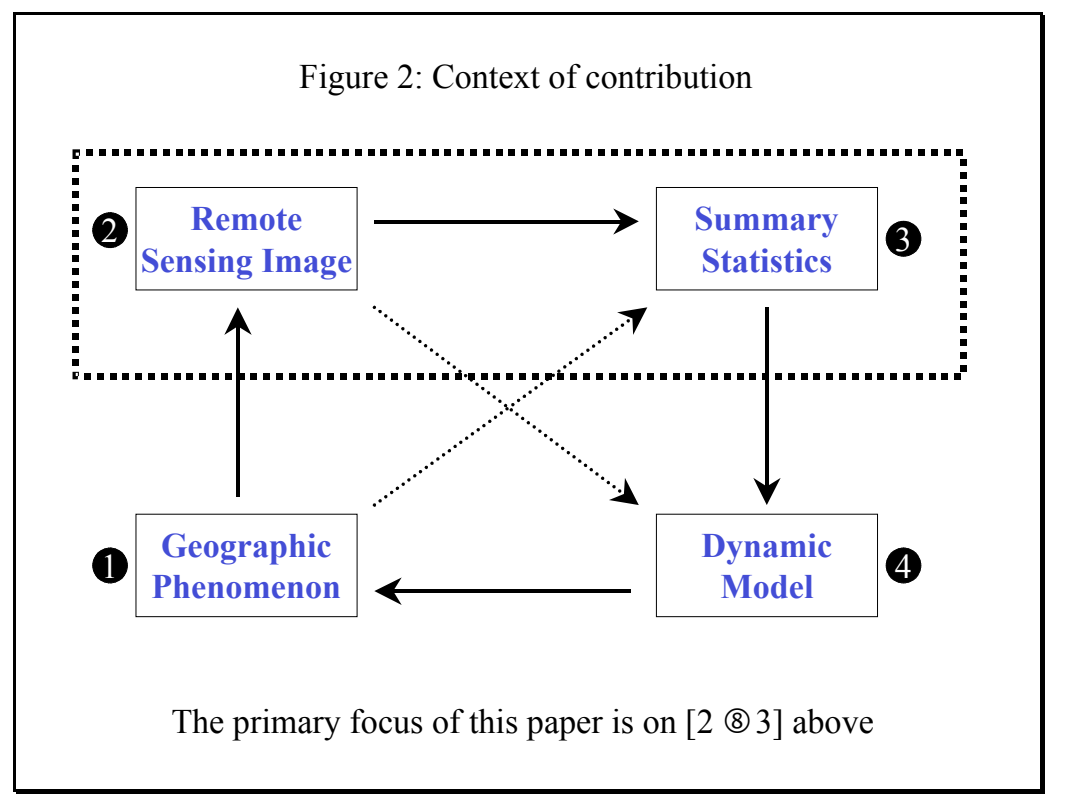

Much work has also been done in the interpretation of remote sensing sensing images of urbanization, a focus that may be represented as $[1 \rightarrow 2]$ in the context of figure 2. Remote sensing image data have been used to detect land use change in China by Yeh (2001) and by Li and Yeh (1998) applying and extending methods introduced by Howarth (1986) and by Mesev, Longley, Batty, and Xie (1995). The [1 $\rightarrow 2]$ designation also includes fuzzy classification 
techniques as applied to the production of remote sensing images. As noted earlier, the method proposed in this work takes a remote sensing image as its starting point and develops summary statistics derived from those data. In the context of figure 2 , therefore, our focus is on [ $2 \rightarrow 3$ ] rather than $[1 \rightarrow 2]$.

In this regard, our work has a similar focus to Anthony Yeh's (2001) recent application of entropy measures derived from remote sensing data to analyze urban growth in the Pearl River. Our work differs from Yeh's primarily with regard to the fuzzy set representation and interpretation of data, and also with regard to our simultaneous derivation and presentation of three urbanization measures derived from a single conceptual framework. As this [2 $\rightarrow 3]$ segment is the immediate object of our inquiry we shall save a more in-depth discussion of this topic for the next section.

More often, summary statistics about urbanization are derived without reference to or mediation by remote sensing image data. In the context of figure 2 we represent such efforts to summarize urbanization levels as a direct $[1 \rightarrow 3]$ path, rather than the indirect $[1 \rightarrow 2] \&[2 \rightarrow 3]$. Most typical of such efforts are jurisdiction-based approaches that seek to define urban or rural territories or population based on the jurisdictions in which they are located. In essence, this approach defines urban territory (or population) as territory (or population) found within urban jurisdictions. Although this circular reasoning is highly unsatisfactory from a theoretical perspective, it is the most common source of official statistics available on urbanization, and it reflects an administrative orientation that is pre-occupied with fiscal and governance issues. These are certainly important issues, but for our purposes a fixation on jurisdictional boundaries tends to obfuscate the nature of the underlying geographic phenomenon.

This is not only a problem in China. The United States Census ${ }^{1}$, for example, also defines the urban-rural dichotomy in circular terms (our emphases below):

"Urban" consists of territory, persons, and housing units in:

- $\quad$ Places of 2,500 or more persons incorporated as cities, villages, boroughs (except in Alaska and New York), and towns (except in the six New England States, New York, and Wisconsin), but excluding the rural portions of "extended cities".

- Census designated places of 2,500 or more persons.

- Other territory, incorporated or unincorporated, included in urbanized areas.

\footnotetext{
${ }^{1}$ The definition is found in U.S. Census Bureau, Urban and Rural Definitions, October 1995.
} 
Territory, population, and housing units not classified as urban constitute "rural".

While that definition may be functional at the level of Census Bureau operations, it does not shed light on the geographical phenomenon we are addressing. Equally unsatisfactory for our purposes is the standard textbook definition: "To an urban economist, a geographical area is considered urban if it contains a large number of people in a relatively high population density" (Sullivan, 1990, p.6) In both instances, place is given a priori, usually in jurisdictional terms, whereas our approach seeks to uncover urban places inductively and empirically.

The challenge in China is no less vexing, as evidenced by Gregory Eliyu Guldin's (1992, p. 5) plaint:

"Never mind then that Rong Ma ... makes a convincing argument for the superiority of a sociological view of urban community as the key to defining towns or that Kam Wing Chan argues against simplistic [dichotomous] categories. Forget that Ma and others ... have pointed out the anomolies of the "town" (zhen) classification and its relationship to the varieties of county, district (qu), xiang and village towns, so the the "town" as encountered in Chinese statistical tables is neither an unchanging category nor a sociologically accurate one. Forget too that Clifton Pannell ..., Graham Johnson ..., and I argue that there are far too many interconnections among all areas of Chinese society simply to bifurcate the society into "rural" and "urban" spheres. Forget all these arguments for social scientifically-based categories or understandings, for in the end all of these must yield to the statistical tables of the State Statistical Bureau and its categories."

Thus, in both the United States and in China, the official state statistics used to record levels of urbanization are fixated on exogenously determined place boundaries, and those places are then classified as either urban or rural based on varying criteria. While such jurisdictional boundaries are of course necessary for effective administration of territories, they are no substitute for (and, indeed, tend to obscure) a direct examination of the underlying geographic phenomena associated with peri-urbanization.

Summary or descriptive statistics of urbanization are not so much ends in themselves; rather, they provide important feedback for land use planners and other decision-makers who may seek to intervene in ways that might generate more favorable future outcomes. Li and Yeh's (1998) and Yeh and Li's (1999) research on the Pearl River Delta region are representative of studies that generate summary statistics from remote sensing data to monitor land use change. This is a direct 
$[2 \rightarrow 3$ ] link in terms of figure 2 , with an implicit [ $3 \rightarrow 1$ ] feedback link to policies regulating the form and extent of urban development. Other works develop and apply dynamic models to complete the feedback loop more explicitly. Zhou and Ma (2000), for example, use summary data to support a descriptive model of economic restructuring and suburbanization in China. In the context of figure 2 their work is best represented by $[4 \rightarrow 1]$ supported by $[1 \rightarrow 3] \&[3 \rightarrow 4]$, as is the work by Zhai and Ikeda (2000). The latter adapt a mathematical ecology model with differential equations that are fed by summary data on urban land use density and other ecological indicators. Another interesting variant of dynamic model is the cellular automatum as applied to urbanization by Batty and Xie (1994) and as applied to the Chinese urbanization context by Li and Yeh (2000). Cellular automata are fed directly by raster data, thereby bypassing the need for summary statistics and so can be represented in figure 2 by [ $\rightarrow \rightarrow 1$ ] supported directly by [1 $\rightarrow 2$ ] $\&[2 \rightarrow 4]$.

The brief sketch above by no means substitutes for a comprehensive review of China urbanization studies. It is, nonetheless, useful for clarifying where our particular contribution fits in within the larger scheme of things. Specifically, we focus on the link between [2 $\rightarrow 3]$ as supported by $[1 \rightarrow 2]$, to produce a meaningful set of summary statistics derived from remote sensing data of peri-urbanizing regions. We argue that such remote sensing images may be interpreted as fuzzy set data that reflect an underlying process of fuzzy urbanization, and we turn now to a more detailed description of the method by which such summary data may be derived.

\section{Fuzzy urban sets}

\section{Fuzzy urban sets}

Consider the set $U$ and all possible members of $U, \mathrm{x}_{\mathrm{i}} \square X$, drawn from some universal set $X$, where i $\square N=\{1,2, \ldots, \mathrm{n}\}$. In classical set theory, membership in $U$ is unambigously or "crisply" defined so that any element $\mathrm{x}_{\mathrm{i}}$ of the universal set $X$ is either a full member of $U$, or not a member at all. This bimodal characteristic of crisp sets is reflected in a membership function for $U, \mathrm{~m}_{\mathrm{U}}($.$) , that only admits two possible values, zero or one:$

$$
\mathrm{u}_{\mathrm{i}}-\mathrm{m}_{U}\left(\mathrm{x}_{\mathrm{i}}\right) \square\{0,1\} \quad \square \mathrm{i} \square N
$$

For many purposes, including our own, this classical bimodal set membership function is unnecessarily restrictive, and so this motivates the introduction of fuzzy sets. Zadeh (1965) 
accomplished this simply by extending the membership function to map from $X$ to the entire unit interval, so that

$$
\mathrm{u}_{\mathrm{i}} \mathrm{m}_{\mathrm{U}}\left(\mathrm{x}_{\mathrm{i}}\right) \square[0,1] \square \mathrm{i} \square \mathrm{N}
$$

where equation 1 is now a special limiting case of equation 2. Fuzzy sets allow a continuum of membership values while crisp sets allow only for the two most extreme possibilities: full membership or no membership at all.

Now, consider a remote sensing image such as the one shown in figure 3 , where each pixel $\mathrm{x}_{\mathrm{i}}$ of the image corresponds to a particular parcel (or plot, or chunk) of land, and where the set $X$ corresponds to the image as a whole, or more precisely, to the union of all individual parcels $\mathrm{x}_{\mathrm{i}}$. For each parcel we may in principle assign a degree of membership in the fuzzy urban set $U$ in accordance with the membership function described in equation 2. As will be explained below, the shades of gray in figure 3 represent the degree of membership thus assigned, with white representing full membership and black representing no membership, and with all shades of gray corresponding to a spectrum of values between these two extremes. The resulting set $U$ (and by implication, its rural complement $R$ ) is "fuzzy" in the sense that the classical yes-no membership dichotomy now dissolves into a question of degree or extent - as with desakota regions.

Figure 3: Remote sensing image of Ningbo City

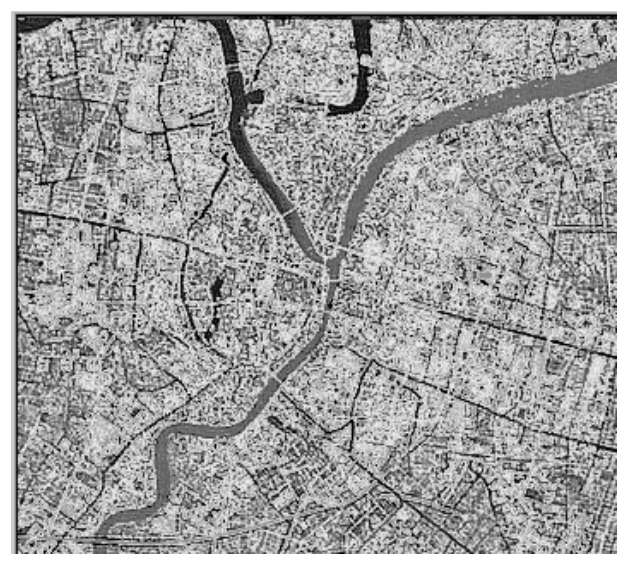

In graphical form, the extension from crispy to fuzzy sets is represented in figure 4, where in this illustration the reference set $X$ (from which all member pixels $\mathrm{x}_{\mathrm{i}}$ are drawn) is a real line 
representation of a 1-D geographical space. Here, the urban-rural dichotomy is transformed into a fuzzier notion of urbanity, where the height of the membership function determines the extent to which a given location $x_{i}$ is a member of the set of all urban locations. If we take $u_{i}=0.5$ as the threshold or cutoff point ${ }^{2}$, then a "crisp" categorization of the space in figure 4 would result in the two crisp urban sets (and by inference, the three crisp rural sets) shown. The urbanized world of today is a crisp one, according to officials statistics on urbanization. The approach advocated here recasts the urban-rural dichotomy in fuzzy terms, with crisp sets as an extreme limiting case.

Figure 4: One dimensional urban sets (fuzzy and crisp)

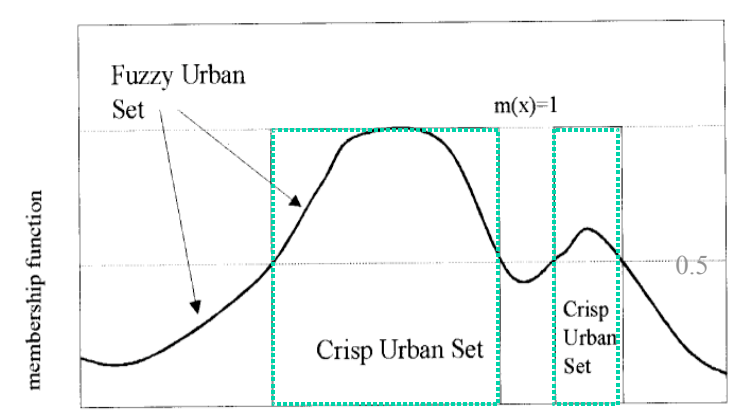

1-D geographic space

This adjustment is significant for several reasons. First, it enables us to introduce ambiguity of land use classification formally and precisely. Fuzziness does not imply imprecision; rather, it implies a more precise way of handling ambiguous land use patterns. Fuzziness is intrinsic to the underlying desakota phenomenon that is being described ${ }^{3}$. Second, it allows us to draw upon and apply work done by Zadeh (1965), Kosko (1997) and others who have contributed to fuzzy set theory, even though their work would appear at first glance to be quite far removed from issues of urbanization in China. For example, let the rural set $R$ be defined as the complement of the urban set $U$, that is $R_{-} U^{\mathrm{c}}$. In the case of crisp sets, the intersection of any set with its complement is null:

2 That is, $\mathrm{x}_{\mathrm{i}}$ is a member of the crisp urban set $U$ whenever $\mathrm{u}_{\mathrm{i}}-\mathrm{m}\left(\mathrm{x}_{\mathrm{i}}\right) \geq 0.5$.

3 Put another way, it is the logic of fuzzy sets, not the fuzzy logic of sets. (This distinction is not recognized in the title to Heikkila's "Fuzzy Logic of Accessibility" paper.) 


$$
U_{-} U^{\mathfrak{c}}=U_{-} R=\varnothing
$$

For fuzzy sets, though, this result no longer applies, and this is potentially significant for how we organize land use data. As another example, Kosko (1997) shows how familiar terms such as entropy can be recast in terms of fuzzy set operations, and we allude to his result in the section that follows. Third, and of most direct import for this paper, casting peri-urbanization patterns as fuzzy urban sets leads directly to the use of three fundamental dichotomies for characterizing urban-rural systems.

\section{Three fundamental dichotomies of fuzzy urban sets}

In a recent paper Heikkila (2000) applies Bart Kosko's (1992) notion of a fuzzy power set to develop three fundamental dichotomies in the context of accessibility ${ }^{4}$. We adapt those same dichotomies here as fundamental descriptors of fuzzy urban sets. The three dichotomies are best understood in the context of Kosko's (1992) graphical depiction of a fuzzy power set, which is depicted here in figure 5. Consider the universal reference set $X=\left\{\mathrm{x}_{1}, \mathrm{x}_{2}, \ldots \mathrm{x}_{\mathrm{n}}\right\}$, which in our case refers to the set of pixels in a remote sensing image, and so $n$ is a very, very large number.

Now consider the power set of $X$, denoted by $2^{X}$, which contains all crisp subsets of $X$. For example, in the three-dimensional case $(n=3)$ which is depicted in figure 5 ,

$$
2^{X}=\left\{\varnothing,\left\{\mathrm{x}_{1}\right\},\left\{\mathrm{x}_{2}\right\},\left\{\mathrm{x}_{3}\right\},\left\{\mathrm{x}_{1}, \mathrm{x}_{2}\right\},\left\{\mathrm{x}_{1}, \mathrm{x}_{3}\right\},\left\{\mathrm{x}_{2}, \mathrm{x}_{3}\right\}, X\right\}
$$

These elements of $2^{X}$ (which are themselves sets) constitute the vertices of an n-dimensional hypercube such as the one in figure 5. This hypercube (including its interior) is the fuzzy power set of $X$. Where the crisp power set is a zero-volume lattice-work of points in n-space, the corresponding fuzzy power set is full-volumed. All points contained within or on the frame defined by the power set represent the universe of possible fuzzy sets for the reference set $X$. Using this representation, following Kosko (1997), the fuzzy urban set $U$ can be depicted as a

\footnotetext{
${ }^{4}$ In "Fuzzy Logic of Accessibility", geographical accessibility is cast as a special case of membership in fuzzy clubs, which in turn are extensions of Tieboutian municipalities. Please refer to Heikkila (2000) for a more extensive discussion.
} 
point within a fuzzy hypercube. Its location with respect to each vertex is specified by the corresponding membership value. For example, in figure 5 the coordinates of $U$ are given as ${ }^{5}$ :
$\mathrm{u}_{1}=\mathrm{m}_{\mathrm{U}}\left(\mathrm{x}_{1}\right)$

$$
\mathrm{u}_{2}=\mathrm{m}_{\mathrm{U}}\left(\mathrm{x}_{2}\right)
$$$$
\mathrm{u}_{3}=\mathrm{m}_{\mathrm{U}}\left(\mathrm{x}_{3}\right)
$$

Figure 5: Kosko's fuzzy hypercube



At this juncture it is useful to pause and consider the situation depicted in figure 5. By representing a desakota region (or its encapsulation in a remote sensing image with $\mathrm{n}$ pixels) as a fuzzy urban set $U$, we can then map $U$ into a single point within Kosko's fuzzy hypercube of dimension $\mathrm{n}$. The key to our approach at measuring urbanization is addressed through a simple question: where does this single point representation of the fuzzy set U lie within the fuzzy hypercube that contains all possible fuzzy urban sets of dimension $n$ ? Looking at figure 5 , three aspects of U's location immediately present themselves:

i. How far is $U$ from the origin $\varnothing$ ?

ii. How far is $\mathrm{U}$ from the midpoint $M$ ?

iii. How far is $\mathrm{U}$ from the principal diagonal $\left[\varnothing_{-} I\right]$ ?

5 Here, for obvious reasons, our rendering of the fuzzy hypercube is limited to three dimensions, which in turn corresponds to a remote sensing image of only three pixels. The reader is asked to bear in mind at all times that our discussion is geared to the $\mathrm{n}$-dimensional case where $\mathrm{n}$ is very large. 
The answers to these three very straightforward locational questions in the context of figure 5 relate directly and intuitively to three corresponding measures of urbanization that when taken together are especially well suited to characterizing desakota phenomena. Expanding upon Heikkila (2000) and Kosko (1997), we characterize the location of the fuzzy urban set $U$ in terms of three fundamental dichotomies as summarized in table 1:

Table 1: Three fundamental dichotomies of fuzzy urban sets

\begin{tabular}{|l|l|l|l|}
\hline $\begin{array}{l}\text { Nature of the } \\
\text { dichotomy }\end{array}$ & $\begin{array}{l}\text { Geographic } \\
\text { interpretation }\end{array}$ & Fuzzy set interpretation & $\begin{array}{l}\text { Geometric interpretation } \\
\text { (figure 5) }\end{array}$ \\
\hline Urban-rural & $\begin{array}{l}\text { Aggregate level of urbani- } \\
\text { zation for the study area }\end{array}$ & $\begin{array}{l}\text { Measure of the cardinality of } \\
\text { the fuzzy set } U\end{array}$ & $\begin{array}{l}\text { Urban-ness increases with } \\
\text { distance from the origin; } \\
\text { maximum urban content at } I .\end{array}$ \\
\hline Fuzzy-crisp & $\begin{array}{l}\text { Extent of desakota } \\
\text { phenomenon }\end{array}$ & $\begin{array}{l}\text { A measure of the fuzziness } \\
\text { of the set } U\end{array}$ & $\begin{array}{l}\text { Fuzziness decreases with } \\
\text { distance from midpoint } M, \text { zero } \\
\text { fuzziness at the vertices. }\end{array}$ \\
\hline Entropy-order & $\begin{array}{l}\text { Diffusion of urbanization } \\
\text { process }\end{array}$ & $\begin{array}{l}\text { Uniformity of membership } \\
\text { of the set } U\end{array}$ & $\begin{array}{l}\text { Maximum entropy along central } \\
\text { diagonal; zero entropy at base } \\
\text { vertices. }\end{array}$ \\
\hline
\end{tabular}

Urban-rural dichotomy. This is a measure of the aggregate level of urbanization for the sample area. As such, it corresponds most closely to conventional or official statistics measuring urbanization. A key difference, however, is that each parcel (or pixel) may in principle be partially urbanized. Thus, for example, a study area of one hundred parcels that is sixty percent urbanized may comprise:
a) sixty urban parcels and forty rural parcels, or
b) one hundred parcels, each of which is sixty percent urban and forty percent rural, or
c) any convex combination of (a) and (b).

Conventional measures, such as those based on the Census definitions reported earlier, only allow for type (a) distinctions, where any given place is either urban or rural but not both.

As noted earlier, the vertex $I$ in figure 5 corresponds to a system that is completely urbanized (each pixel, $\mathrm{x}_{\mathrm{i}}$, is a full member of the set $U$ ). At the other extreme, the vertex $\varnothing$ corresponds to a completely rural system, where each pixel is a full member of $R=U^{\mathfrak{C}}$, defined as the complement of $U$. In the context of figure 5, therefore, the urban-rural dichotomy is measured in terms of the distance of $U$ from $\varnothing$. This measure is operationalized most simply by taking the mean value of 
$U$ 's base vectors, the resultant value of which is automatically normalized to the zero-one interval $^{6}$ :

$$
\mathrm{A}(U)-\square_{\mathrm{i}} \mathrm{u}_{\mathrm{i}} / \mathrm{n}
$$

Fuzzy-crisp dichotomy. This interpretation is rooted in the potentially ambiguous nature of fuzzy set membership and, as has been explained above, corresponds most directly to the fuzziness or ambiguity inherent in desakota land use patterns. The height of ambiguity (ie, maximum fuzziness) occurs at the midpoint $M$ in figure 5 where each parcel of land has a 0.50 degree of membership in the urban set $U$ and a 0.50 degree of membership in the rural set $R$. In contrast, each vertex of the fuzzy power set corresponds to one of the crisp subsets of $X$. Conventional methods for measuring urbanization restrict our possibilities to the vertexes alone, while the approach proposed here opens up the interior spaces as well, all of which retain some degree of fuzziness. In the context of urbanization, the midpoint $M$ in figure 5 corresponds to the ultimate desakota condition, where urban is rural and where rural is urban. The complement $U^{\mathfrak{c}}$ of a perfectly fuzzy urban set is a perfectly fuzzy rural set. ${ }^{7}$ Kosko (1997) operationalizes this measure of fuzziness ${ }^{8}$ using fuzzy set operators:

$$
\mathrm{F}=\mathrm{c}\left[U_{-} U^{\mathrm{c}}\right] / \mathrm{c}\left[U \quad \mathrm{U} U^{\mathrm{c}}\right]
$$

where $\mathrm{c}(U)=\square_{\mathrm{i}} \mathrm{u}_{\mathrm{i}_{-}}$is a measure of the count or cardinality for a set, and where the intersection and union of two fuzzy sets are given by the pairwise minima and maxima, respectively. Note that when $U$ is crisp (corresponding to any of the vertices in figure 5), fuzziness drops to zero while fuzziness equals one at the midpoint.

Entropy-order dichotomy. Any point along the principal diagonal of the fuzzy hypercube in figure 5 represents a state of perfectly uniform diffusion of urbanization throughout the study area. The opposite state would be one of complete concentration whereby all urbanization is concentrated in a single spatial unit. In terms of figure 5, the perfect concentration case corresponds to one of the base vectors: $(1,0,0),(0,1,0)$, or $(0,0,1)$. These base vectors provide the

\footnotetext{
6 Another alternative would be to take the root mean square of the base vector values.

7 Here we are using the term "rural" as an all-encompassing "non-urban" category.

${ }^{8}$ Kosko (1997) refers to this measure of fuzziness as fuzzy mutual entropy. See text below for further discussion.
} 
sharpest angle away from the principal diagonal. All other points within the fuzzy cube including $(1,1,0),(1,0,1)$, and $(0,1,1)$ - correspond to some intermediate level of concentration or dispersion. It is clear, therefore, that the principal diagonal corresponds to perfect diffusion, and that the angle away from this diagonal is a measure of spatial concentration.

Batten (1983, chapter 2) provides a clear-sighted review of the concept of entropy and its applications to spatial analysis. Ironically, the very meaning of "entropy" has become highly diffused among myriad applications and interpretations. For our purposes, the specific measure of entropy we use evolves from the work of C. E. Shannon (1948) and Henri Theil (1967). Theil adapted Shannon's entropy statistic for use as an inverse measure of industrial concentration. A similar measure was quickly adapted for use by geographers intent on measuring the degree of concentration for spatial phenomena.

In the context of urbanization studies entropy measures the dispersion of observed urban development patterns. For example Yeh (2001), in his recent study of urban sprawl in the Pearl River Delta region of Southeast China, uses Theil's entropy measure as modified by Thomas, (1981).

$$
\mathrm{E}=\square_{\mathrm{i}} \mathrm{p}_{\mathrm{i}} \log \left(1 / \mathrm{p}_{\mathrm{i}}\right) / \log (\mathrm{n})
$$

where $p_{i}$ measures the proportion of development occuring in a particular zone. For our purposes $p_{i}=u_{i} / \square_{i} u_{i} .9$ This measure of entropy reaches its maximum of one when development is uniformly distributed across the study area (so that $\mathrm{p}_{\mathrm{i}}=1 / \mathrm{n}, \square \mathrm{i} \square N$ ), and it is minimized at zero when all development is concentrated in one location $j$ (so that $p_{j}=1$, and $p_{i}=0, \square i \neq j$ ). Thus, in the context of figure 5 , the central $[\varnothing-I]$ diagonal represents perfect entropy $(E=1)$, and entropy decreases with the angle away from this central diagonal, with zero entropy occuring at any of the base vertices.

Notwithstanding its similar mathematical structure, the spatial concentration measure above differs from another another entropy measure rooted in information theory and originating from Shannon (1948). From the information theory perspective, each pixel contains a signal, and

\footnotetext{
9 Yeh uses density of development as the underlying variable. From our perspective, development density
} is a reasonable basis for measuring membership in $U$, and so the two approaches are basically consistent. 
Shannon's entropy statistic S measures the information content of a set of signals ${ }^{10}$. If all pixels contain the same signal, $\mathrm{S}$ is zero. This is precisely contrary to the interpretation commonly used in studies of geographic dispersion as discussed above, and this has no doubt contributed to general confusion about the use of the term "entropy". Both Shannon's information statistic S and the geographic disperson statistic $\mathrm{E}$ used here are measures of dispersion. The key distinction is that the geographic measure of entropy is concerned with dispersion over spatial units while the information statistic is concerned with dispersion over the potential range of signals.

Thus, we note that entropy in a spatial dispersion context has a distinct interpretation. Indeed, Batty (1974) introduced a measure of spatial entropy to accommodate the discrete nature of space. Batty's measure incorporates a term $\square \mathrm{x}_{\mathrm{i}}$ which provides a measure of the interval over which the corresponding probabilities are defined. Thus, we have

$$
\mathrm{B}=-\square_{\mathrm{i}} \mathrm{p}_{\mathrm{i}} \log \left(\mathrm{p}_{\mathrm{i}} / \square \mathrm{x}_{\mathrm{i}}\right) / \log (\mathrm{n})
$$

In our case, each pixel is of the same size, and so $\square \mathrm{x}_{\mathrm{i}}=\square \mathrm{x}$ for all $i$. In this case $\mathrm{B}$ reduces to

$$
\mathrm{B}=\log (\square \mathrm{x}) / \log (\mathrm{n})+\mathrm{E}
$$

and so the additional term becomes a non-varying shift operator. As noted by Shannon (1948) and others, a measure of entropy is arbitrary subject to any tranformation that preserves order, so we are free to choose spatial units as we please. As each pixel is a standardized spatial unit, a logical choice is $\square \mathrm{x}=1$, in which case Batty's spatial entropy measure reduces to the one we are using. In applications where spatial units may vary, it will be important to replace equation 8 with equation 9.

A final observation regarding entropy before we move on. As noted in footnote 7 above, Kosko presents a measure of fuzzy mutual entropy that is different yet again from any of the entropy measures discussed thus far. His is derived from Kullback's (1959) measure of information gain

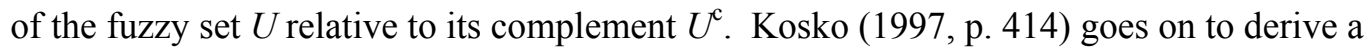
relationship whereby "fuzzy mutual entropy equals the negative of the divergence of Shannon

\footnotetext{
${ }^{10}$ Shannon's entropy measure is $S=\square_{i} p_{i} \log \left(1 / p_{i}\right)$, which ranges from 0 to $\log n$, hence the normalization in equation 8. As noted in the text, the summation operator in Shannon's measure ranges over all possible signals, while the summation operator in equation 8 ranges over all spatial units.
} 
entropy", and where "we can in principle recover Shannon entropy ... from fuzzy mutual entropy by integrating". The interpretation of this relationship in the context of desakota urbanization is beyond the scope of this paper, except to note that equation 7 is in fact Kosko's fuzzy mutual entropy measure ${ }^{11}$.

\section{What the fuzzy cube hath wrought}

Taken as an ensemble, these three dichotomies provide a highly comprehensive characterization of the status and scope of urbanization for a study area. Not all of the measures derived from this conceptual device are new. For example, the aggregate measure of urbanization defined by equation 6 corresponds fairly directly to conventional measures. Even here, however, the interpretation of the statistic as a measure of cardinality of the fuzzy set $U$ is unique, and accomodates varying degrees of urbanity within each spatial unit. Likewise, the measure of entropy used here is not new, but the fuzzy hypercube context makes clear which entropy measure is most appropriate for this application. The fuzziness measure in equation 7 is new to urbanization studies, however, as it is a direct measure of the fuzziness of the urban set U. As noted already, this fills a void in the literature on desakota type urbanization formations. Over the past decade scholars have increasingly recognized the significance and extent of this phenomenon, but have been unable to measure it. Our approach fills this void.

From a theoretical perspective, the main contribution of the fuzzy urban set formulation is the introduction of a unifying conceptual framework for measures of urbanization. This is made possible by the representation of an entire study area as a single point within a fuzzy hypercube. Our three measures of urbanization are derived through a very intuitive process of identifying the location of that point. Extensions of this conceptual device come readily to mind; for example, one may envision the evolution of a study area over time in terms of the trajectory described by the fuzzy set $U$ as it moves within the confines of the fuzzy hypercube that contains it. A focus for research on urban development would then be to articulate an understanding of how fuzzy urban sets evolve in this context.

\footnotetext{
${ }^{11}$ Our geometric interpretation also is indicative of a relationship between these two distinct measures of entropy - F (Kosko's fuzzy mutual entropy) and E (Shannon's entropy) - given the aggregate extent of urbanization, A. E is a measure of the angle away from the principal diagonal. A is a measure of the distance from the origin. Taken together they tell us how far away we are from the midpoint, and that in turn is a measure of $\mathrm{F}$.
} 


\section{Application to Ningbo, China}

We contend that the three measures operationalized in equations 6,7 , and 8 provide a comprehensive set of urbanization statistics well suited to measuring, monitoring, characterizing, and comparing fuzzy urban sets. As remote sensing data become increasingly available urbanization researchers will become less dependent upon jurisdiction-bound statistics that are illsuited to conveying the contours of desakota phenomenon, and so we envision significant scope for widespread adaptation of this approach. Moreover, as the volume of remote sensing data increases, the need for meaningful summary statistics will become more acute. Our approach responds directly to that need.

To test the feasibility of our proposed method and approach we turn now to a pilot application. The study area we have selected is Ningbo City, Zhejiang Province, in eastern China south of Shanghai. Ningbo is an active port area and an industrial center on the Yong River. Located in a fertile agricultural region, it is well known for its rural industry. ${ }^{12}$ This case study was selected in part because of the rapid and diffuse patterns of urbanization found there and, in part, because of data availability.

\section{Generating membership values for the fuzzy urban set $U$}

Recall from our discussion of figure 2, that the starting point for our method is the remote sensing image. Of course, in practice, considerable effort is required to reach the starting point. The basic issue is how to generate a gray-scaled membership value $\mathrm{u}_{\mathrm{i}}$ for each pixel $i$, indicating it's degree of membership in the fuzzy urban set $U$. The method described in the preceeding pages presumes the existence of these membership values and proceeds forward from there. Indeed, this paper makes no claim of any original scholarly contribution regarding the interpretation of remote sensing images. Instead, we use fairly conventional remote sensing image classification methods to move from $[1 \rightarrow 2]$. This section briefly describes the process by which we generated a set of membership values $u_{i}$ for the fuzzy urban set depicted in figure 3 .

Remote sensing data are generated by measuring the percentage reflection of electromagnetic radiation across selected spectral bands from the electromagnetic spectrum. Remote sensing data sample three discrete spectral bands, and so each pixel in our dataset has a (percentage reflection) datum associated with each of the three bands. Our test case uses Landsat TM2, TM5, and TM7

\footnotetext{
${ }^{12}$ See http://www.ningboport.com/
} 
bands for three points in time; 1987, 1990, and 1996; each with 30m x 30m resolution and a 350 x 312 pixel image. As with most remote sensing applications, our approach is geared to interpretation of land cover. Of course, the extent to which a spatial unit is urbanized will be determined by other considerations beyond land cover. Factors such as population density, building heights, or interaction variables are often cited. Increasingly, therefore, remote sensing applications are being integrated with GIS in order to incorporate such considerations. We do not do so here for two reasons. First, our intent is to demonstrate the feasibility of moving from $[2 \rightarrow$ $3]$, in the context of figure 2 for a given set of membership values. For this purpose, any reasonable set of membership values $u_{i}$ will suffice. Secondly, we envision that our proposed method will be useful for compiling urbanization statistics on a global scale. Remote sensing data are increasingly available at that scale while GIS data are not.

We employ three steps to generate a set of membership values $u_{i}$. First, a supervised classification procedure is used to classify the remote sensing data into five separate categories corresponding to greenbelt, water, roads, rural, and urban using Bayer's classification method as described by Campbell (1996, chapter 11). Secondly, pixels falling initially into the greenbelt, water, or roads categories are assigned grey values along the initial urban-rural spectrum based on the values of neighboring pixels (see Richards and Jia, 1999). In the third and final step, the Bayer's classification method is reapplied, and values of the resultant discriminant function are used to derive the degree of membership $u_{i}$ of each pixel. This brings us to the "starting point" for our method $[2 \rightarrow 3]$.

\section{Calculating fuzzy urban set statistics for study area}

Our method for moving from [ $2 \rightarrow 3]$ is simply to calculate the three measures corresponding to equations 6,7 , and 8 respectively. Doing so yields the following results for our Ningbo City study area:

Table 2: Ningbo City as a fuzzy urban set

\begin{tabular}{|l|l|l|l|}
\hline Year & Level of urbanization & Extent of fuzziness & Degree of Entropy \\
\hline $\mathbf{1 9 9 6}$ & 0.557 & 0.366 & 0.723 \\
\hline $\mathbf{1 9 9 0}$ & 0.553 & 0.365 & 0.572 \\
\hline $\mathbf{1 9 8 7}$ & 0.584 & 0.380 & 0.620 \\
\hline
\end{tabular}


The results point to the following conclusions:

- The measured level of urbanization in the study area fell perceptibly from 1987 to 1990, but remained essentially unchanged from 1990 to 1996. In the context of figure 5 this implies movement towards the origin $\varnothing$.

- The fuzziness of the urbanizing system decreased over time. This tells us that land use patterns are beginning to articulate themselves more distinctively, with clearer demarcations between urban and rural land uses. In the context of figure 5, it denotes movement away from the midpoint $\mathrm{M}$ and towards one of the outer vertices.

- The level of entropy within the system varies markedly between periods within the range of 0.57 to 0.72 . In the context of figure 5 this tells us that the urban set $U$ is located somewhere near the central diagonal.

Taken together, these results tell us that the representation of the Ningbo City study area in the context of Kosko's fuzzy hypercube is not far from the central diagonal, moving slowly away from $M$ and towards the origin. The cumulative impression given by these data is of a study area wherein the distinctions between urban and rural are sharpening while urbanization itself is less intense but more spatially dispersed.

This pilot case demonstrates several useful points. First, and most importantly, it demonstrates the feasibility of the general approach advocated here. However, it also underlines the importance of continuing research in the classification of remote sensing data, for the results of any procedure [2 $\rightarrow 3]$ to derive summary statistics from the membership data will depend critically on the quality of those data and of the procedures $[1 \rightarrow 2]$ used to derive them. For example, a sequence of time series images may reflect differences in weather conditions rather than underlying changes in urbanization. This is a problem that is well recognized in the remote sensing field, but we iterate the point here. More generally, just as a rising tide lifts all ships, continuing remote sensing methodological improvements will enhance the quality of the summary statistics proposed here. 


\section{Concluding Remarks}

From the perspective of the Chinese government, continued urbanization is a top priority for China's economic development and "a symbol of continuous human progress and the historical trend of the contemporary world". ${ }^{13}$ At present, just over thirty percent of China's population is regarded as urban, compared to less than twenty percent in 1978. With a total population of 1.2 billion and growing, the implications of aggressive urbanization in China are staggering. The State Development Planning Commission, the Ministry of Construction, the World Bank, and many other national and international agencies are struggling to help formulate policies to facilitate and promote effective urbanization ${ }^{14}$. A central question in this regard concerns the appropriate spatial form and distribution of urbanization. Although urban geographers are well aware of the prevalence and significance of desakota urbanization patterns in China and elsewhere, urban economists have been much less inclined to focus on this phenomenon. One reason for this reluctance is that spatially complex diffusion patterns cannot be summarized or characterized succinctly, and therefore are not readily incorporated into the kinds of formal models that economists are accustomed to using. As we have seen, urban geographers are moving more quickly from descriptive narratives of desakota phenomena to a range of computerbased modeling approaches. However, even in the absence of formal models, summary data are important for our ability to grasp the evolving nature and extent of urbanization in China and elsewhere.

The method proposed in this paper addresses this challenge in a unique way. The approach is at once simple yet comprehensive. By formulating an urbanizing region (or a remote sensing image thereof) as a fuzzy urban set, we can characterize its development in terms of three fundamental dichotomies: (i) urban-rural, (ii) fuzzy-crisp, and (iii) entropy-order. Moreover, these three measures each stem from a single geometric interpretation whereby the fuzzy urban set $U$ is located as a single point within Kosko's fuzzy hypercube. A comprehensive characterization of the observed urbanization phenomenon is derived simply by describing the fuzzy set $U$ within this unifying context. This provides a solid basis for comparing urbanization patterns over time and in different places, and it provides succinct measures of urbanization that may in turn support the development of new classes of urbanization models.

\footnotetext{
${ }^{13}$ See remarks by Zhao Baojiang, Vice Minister of China's Ministry of Construction at World Bank (2000) workshop on urbanization in China.

${ }^{14}$ See Chan (1992) or Ma and Fan (1994) for a contemporary historical perspective.
} 
Having said this, many issues remain to be addressed before this approach can be adapted widely and systematically. Foremost among these is the question of scope and scale of the areas to be compared. The measured attributes of any given study area will vary with one's choice of the corresponding remote sensing image boundary or resolution, and it is difficult to avoid a certain element of arbitrariness in this selection. Regarding boundary issues, one practical approach is to work within existing jurisdictional boundaries and measure urbanization trends within this framework. An advantage of this approach is that it provides a frame of reference which, albeit arbitrary, has meaningful links to decision-making. This is less useful, however, wherever the focus of urbanization extends across jurisdictional boundaries, as is increasingly the case. Another disadvantage of using jurisdictional boundaries is the pitfall of circular reasoning alluded to earlier whereby urban places are defined, in effect, as exogenously defined places that meet some criteria for "urban". Working with fuzzy urban sets softens this dilema somewhat by allowing degrees of urbanity, but it does not address the issue of place definition in a more fundamental geographic sense. ${ }^{15}$ Even if place (or remote sensing image) boundaries were agreed upon, one's measure of urbanization is likely to depend upon the resolution of the image itself.

Of course, these issues are not new, nor are they specific to the fuzzy urban set approach advocated here, but they are issues that must be contended with before our proposed method can be subject to systematic and widespread use. Notwithstanding these challenges, we aver that fuzzy urban sets are a useful paradigm for characterizing urbanization trends in China and other regions where desakota patterns are the norm.

\footnotetext{
${ }^{15}$ A more promising approach may be to define an urban place more endogenously in cartographic terms as an aggregation of urban pixels, although this too invites many more questions regarding spatial autocorrelation, contiguity, etc.
} 


\section{References}

\section{Batten, David F. (1983)}

Spatial Analysis of Interacting Economies, Kluwer-Nijhoff Publishing; Boston, The Hague, and London.

\section{Batty, Michael (1974)}

Spatial Entropy, Geographical Analysis, vol. 6, 1-31.

\section{Batty, Michael and Y. Xie (1994)}

From Cells to Cities, Environment and Planning B, vol. 21, 531-548.

\section{Campbell, James (1996)}

Introduction to Remote Sensing, $2^{\text {nd }}$ edition, Guilford Press, New York.

\section{Chan, Kam Wing (1992)}

Economic Growth Strategy and Urbanization Policies in China, 1949-1982, International Journal of Urban and Regional Research, vol. 16(2), 275-305.

\section{Guidin, G. E. (1997)}

Desakotas and Beyond: Urbanization in Southern China. In Farewell to Peasant China: Rural Urbanization and Social Change in the Late Twentieth Century, edited by G.E. Guldin. Armonk, New York., London, England: M.E.Sharp. 47-67

\section{Heikkila, Eric J. (2000)}

The Fuzzy Logic of Accessibility, in Donald G. Janelle and David C. Hodge, editors, Information, Place, and Cyberspace: Issues in Accessibility, Springer, Berlin, 91-106.

\section{Howarth, P. J. (1986)}

Landsat Digital Enhancements for Change Detection in Urban Environment, Remote Sensing of Environment, vol 13, 149-160.

\section{Jensen, John R. (1996)}

Introductory Digital Image Processing: A Remote Sensing Perspective, $2^{\text {nd }}$ edition, PrenticeHall, Upper Saddle River, NJ.

Kosko, Bart (1997) 
Fuzzy Engineering. Upper Saddle River, NJ: Prentice Hall.

\section{Kosko, Bart (1992)}

Neural Networks and Fuzzy Systems: A Dynamic Systems Approach to Machine Intelligence.

Englewood Cliffs, NJ: Prentice Hall.

Kullback, S. (1959)

Information Theory and Statistics, New York: Wiley.

\section{Lee, Yok-Shiu (1991)}

Rural Nonagricultural Development in an Extended Metropolitan Region: The Case of Southern Jiangsu. In The Extended Metropolis: Settlement Transition in Asia, edited by N. Ginsburg, B.Koppel, T.G. McGee. Honolulu: University of Hawaii Press. 137-156.

\section{Li, Xia. and A. Yeh (2000)}

Modelling sustainable urban development by the integration of constrained cellular automata and GIS, International Journal of Geographic Information Science, vol. 14(2), 131-152.

\section{Li, Xia. and A. Yeh (1998)}

Principal Component Analysis of Stacked Multi-temporal Images for Monitoring Rapid Urban Expansion in the Pearl River Delta, International Journal of Remote Sensing, vol. 19(8), 1501-1518.

\section{Laurence J. Ma and Ming Fan (1994)}

Urbanization from Below: The Growth of Towns in Jiangsu, China, Urban Studies, vol. 31(10), 1625-1645.

\section{McGee, Terry (1991)}

The Emergence of Desakota Regions in Asia: Expanding a Hyopthesis. In The Extended Metropolis: Settlement Transition in Asia, edited By N. Ginsburg, B.Koppel, T.G. McGee. Honolulu: University of Hawaii Press. 3-25.

\section{Mesev, T.V., P.A. Longley, Batty, M. and Y. Xie (1995)}

Morphology from Imagery: Detecting and Measuring the Density of Urban Land Use, Environment and Planning A, vol. 27, 759-780.

\section{Richards, John A. and Xiuping Jia (1999)}


Remote Sensing Digital Image Analysis: An Introduction, $3^{\text {rd }}$ edition, Berlin: Springer-

Verlag.

Shannon, C.E. (1948)

A Mathematical Theory of Communication, Bell System Technical Journal, vol. 27, 379-423 and 623-656.

Sullivan, A.M. (1990)

Urban Economics, Homewood, Boston.

Theil, H. (1967)

Economics and Information Theory, North-Holland, Amsterdam.

Thomas, R.W. (1981)

Information Statistics in Geography, Hutchins \& Sons, Norwich.

Wang, F. (1990)

Improving Remote Sensing Image Analysis Through Fuzzy Information Representation, Photogrammetric Engineering \& Remote Sensing, vol. 56(8), 1163-1169.

World Bank (2000)

Proceedings for Workshop on China's Urbanization Strategy: Opportunities, Issues and Policy Options, May $8^{\text {th }}-10^{\text {th }}$, World Bank Office, Beijing.

Yeh, Anthony (2001)

The Measurement and Monitoring of Urban Sprawl in a Rapidly Growing Region Using Entropy, Photogrammetric Engineering and Remote Sensing, forthcoming.

Yeh, Anthony and Li, Xia. (1998)

Economic Development and Agricultural Land Loss in the Pearl River Delta, China, Habitat International, vol. 23(3), 373-390.

Zadeh, Lofti (1965)

Fuzzy Sets, Information and Control, vol. 8, 338-353.

\section{Zhai, Guofang and Saburo Ikeda (2000)}

An Empirical Model of Land Use Change in China, Review of Urban and Regional Development Studies, vol. 12(1), 36-53. 


\section{Zhang, L. and X. B. Zhao (1998)}

Re-examining China's "Urban” Concept and the Level of Urbanization, The China Quarterly, $330-381$.

\section{Zhou, Daming (1997)}

On Rural Urbanization in China, in Farewell to Peasant China: Rural Urbanization and Social Change in the Late Twentieth Century, edited by G.E. Guldin, Armonk, New York:

M.E. Sharpe, 227-247.

\section{Zhou, Yixing (1991)}

The Metropolitan Interlocking Region in China: A Preliminary Hypothesis. In The Extended Metropolis: Settlement Transition in Asia, edited By N. Ginsburg, B.Koppel, T.G. McGee. Honolulu: University of Hawaii Press. P89-111.

\section{Zhou, Yixing and Laurence J. C. Ma (2000)}

Economic Restructuring and Suburbanization in China, Urban Geography, vol. 21(3), 205236. 